\title{
Mapping Netrin Receptor Binding Reveals Domains of Unc5 Regulating Its Tyrosine Phosphorylation
}

\author{
Robert P. Kruger, ${ }^{1,2}$ Jeeyong Lee, ${ }^{1,3}$ Weiquan Li, ${ }^{1}$ and Kun-Liang Guan ${ }^{1,3,4}$ \\ ${ }^{1}$ Life Sciences Institute, ${ }^{2}$ Neuroscience Program, ${ }^{3}$ Department of Biological Chemistry, and ${ }^{4}$ Institute of Gerontology, University of Michigan, Ann Arbor, \\ Michigan 48109
}

\begin{abstract}
Netrin and its receptors Unc5 and deleted in colorectal carcinoma (DCC) regulate axon guidance and cell migration. We defined domains involved in the interactions between netrin-1, DCC, and Unc5c. We show that Unc5 requires both Ig domains to interact with netrin. DCC binds through the fourth fibronectin type III domain, whereas netrin binds through multiple domains to both receptors. We examined the functional consequences of removing the netrin binding and nonbinding domains from Unc5 in vitro and in vivo. In human embryonic kidney 293 cells, removal of the netrin binding second Ig domain causes an increase in basal tyrosine phosphorylation, whereas removal of the netrin nonbinding thrombospondin domains decreases tyrosine phosphorylation. Moreover, experiments in Caenorhabditis elegans indicate that both netrin binding and nonbinding domains are necessary for phenotypic rescue of an unc-5 loss of function mutation.
\end{abstract}

Key words: DCC; Unc5; netrin; axon guidance; receptor phosphorylation; cell migration

\section{Introduction}

Guiding developing neurons to appropriate targets requires a combination of external cues that attract, repel, and signal where to adhere and when to branch. The tip of extending axons, the growth cone, responds to gradients of external factors that direct its motility. Growth cones have receptor proteins that bind to ligands and initiate signals that cause neurons to grow, turn, and stop. Using genetic and biochemical methods, many ligand and receptor pairs have been identified (Dickson, 2002), among them are netrin and its receptors Unc5 and DCC (deleted in colorectal carcinoma).

Netrin is a chemoattractant for commissural neurons in vertebrates (Kennedy et al., 1994) and is homologous to a Caenorhabditis elegans protein, UNC-6, that governs circumferential axon guidance and gonad distal tip cell (DTC) migration (Hedgecock et al., 1990; Ishii et al., 1992). Both Netrin and UNC-6 are attractive to some neurons and repulsive to others (Colamarino and Tessier-Lavigne, 1995). How netrin is interpreted by a given neuron depends on which netrin receptors are expressed. Netrin signals attraction through an interaction with DCC and UNC-40 (Chan et al., 1996; Keino-Masu et al., 1996), whereas repulsion requires another receptor, Unc5 (Wadsworth et al., 1996; Leonardo et al., 1997).

Netrin has the flexibility to signal through DCC alone, DCC

Received May 12, 2004; revised 0ct. 15, 2004; accepted 0ct. 18, 2004.

This work was supported by grants from the National Institutes of Health, the Walther Cancer Institute, a MacArthur Fellowship (K.-L.G.), and fellowship support (R.P.K.) from the Institute of Gerontology (5 T32 G00114). We thank J. Cullotti for pU5HA, E. Fearon for pDCC, the Caenorhabditis Genetics Center for unc-5(e53) worms, and J. Aurandt for critical reading of this manuscript.

Correspondence should be addressed to Kun-Liang Guan, Life Sciences Institute, University of Michigan, 210 Washtenaw Avenue, Ann Arbor, MI 48109. E-mail: kunliang@umich.edu.

DOI:10.1523/JNEUROSCI.3715-04.2004

Copyright $\odot 2004$ Society for Neuroscience $\quad$ 0270-6474/04/2410826-09\$15.00/0 and Unc5 together, or Unc5 alone. Although DCC by itself signals attraction, coexpression of Unc5 converts DCC-mediated attraction to repulsion (Hong et al., 1999). Unc5 has also been shown to signal repulsion independent of DCC (Keleman and Dickson, 2001), indicating that DCC is not absolutely necessary as a coreceptor for Unc5 signaling. Both DCC and Unc5 are type I transmembrane proteins of the Ig superfamily with 10 and 4 extracellular domains, respectively. Netrin, also a multidomain protein, shares homology with laminin. The multiple effects of netrin are initiated through this handful of domains.

To understand how netrin interacts with its receptors and determine how netrin-binding leads to receptor tyrosine phosphorylation, we have analyzed domain deletion mutants of DCC, Unc5, and netrin for their ability to interact in a cell-based assay. We find individual domains in DCC and Unc5 and multiple domains in netrin needed for binding. Using this information we examined the functional consequences of removal of the netrin binding and nonbinding domains in Unc5 in vitro and in $C$. elegans.

\section{Materials and Methods}

Plasmid constructs. pCDNA3-Unc5c with a hemagluttinin (HA) tag was constructed by amplification of Unc-5c (gi:3789764) from a human brain cDNA library followed by subcloning into pCDNA3-HA. pNet(AP) was constructed by PCR amplification of netrin-1 cDNA and SEAP (secreted alkaline phosphatase) cDNA (pSEAP2 vector; Clontech) and subcloning into pCDNA3. Point and deletion mutants were made using the QuickChange site-directed mutagenesis kit (Stratagene, La Jolla, CA) from the following templates: pcDNA3-Unc5c(HA), pNet(AP), pDCC (a gift from E. Fearon, University of Michigan, Ann Arbor, MI), and pU5HA (a gift from J. Cullotti, Mount Sinai Hospital, Toronto, Ontario, Canada). For large deletions, primers annealed to $27 \mathrm{bp}$ on each side of the gap. Sequencing was used to confirm deletions. Human Unc5c deletion constructs are: $\Delta \operatorname{Tsp} 1(\mathrm{Y} 258-\mathrm{P} 315), \Delta \mathrm{Tsp} 2(\mathrm{~V} 316-\mathrm{M} 368), \Delta \mathrm{Tsps}(\mathrm{Y} 258-$ 
M368), $\Delta \operatorname{Ig} 1(E 51-A 163), \Delta \operatorname{Ig} 2(Y 162-V 257)$, and $\Delta \operatorname{Igs}(E 51-V 257)$. Deletion constructs for human DCC are $\Delta \mathrm{FN} 3-6$ (P625-G1031), $\Delta \mathrm{FN} 5$ (T842-S929), $\Delta$ FN5-6(Y809-G1031), $\Delta$ FN3(P625-T715), $\Delta$ FN4(P726-L808), FN46(G54-T715), FN5-6(G54-L808), FN4-5(G54-T715;T945-G1031), and FN4(G54-T715, Y809-G1031). For FN55 and FN44 the deletion boundary of $\Delta \mathrm{FN} 5$ and $\Delta \mathrm{FN} 4$ was converted to an NheI site, into which FN4 (S723T814) and FN5 (S841-A935) were inserted, respectively. Human netrin-1alkaline phosphatase (AP) deletion constructs are: $\Delta$ Lam VI(G46-V281), $\Delta$ Lam V(R284-E467), $\Delta$ C345C(M487-Q594), and C345C ( $\Delta$ G46-E467). C. elegans UNC-5 constructs generated from $\mathrm{pU} 5 \mathrm{HA}$ are $\Delta \operatorname{Igs}(\mathrm{C} 25-\mathrm{N} 188)$ and $\Delta \mathrm{Tsps}(\mathrm{Y} 200-\mathrm{T} 326)$.

Binding assay with netrin-alkaline phosphatase. Netrin-AP was concentrated ( $\sim 500$-fold) using Centriprep centrifugal filtration devices (Millipore, Bedford, MA) from stably expressing human embryonic kidney 293 (HEK293) cells. An alkaline phosphatase assay using paranitrophenylphosphate (pNPP) determined netrin-AP concentration (Vikis et al., 2002). After 36-48 hr in culture, cells transfected with wild-type (wt) or mutant receptor constructs were incubated for $30 \mathrm{~min}$ with 2 nM netrin-AP in Opti-MEM (Invitrogen, San Diego, CA) and 10 $\mu \mathrm{g} / \mathrm{ml}$ heparin to block nonspecific binding (Keino-Masu et al., 1996). Cells were stained for netrin binding as described previously (Vikis et al., 2002). Netrin-AP binding was blocked by pretreating Unc5c- or DCC-expressing cells with $10 \mu \mathrm{g} / \mathrm{ml}$ purified chicken myc-tagged netrin for $15 \mathrm{~min}$.

An on-bead assay was used for quantitative comparison of Unc5c deletion mutant binding to netrin. HA-tagged proteins were immunoprecipitated from transfected HEK293 cells, aliquoted, and incubated with netrin at concentrations ranging from 1.25 to $40 \mathrm{~nm}$. Bound netrin was detected using a pNPPase assay.

To assess cell surface expression, a cell-impermeable biotinylation reagent (EZ-link Sulfo-NHS-LC-Biotin; Pierce, Rockford, IL) was incubated with Cos-7 cells expressing Unc5c or DCC proteins according to the manufacturer's protocol. Cells were lysed with NP-40 buffer [ $20 \mathrm{~mm}$ Tris pH 7.5, $100 \mathrm{~mm} \mathrm{NaCl}, 1 \% \mathrm{NP}-40,5 \mu \mathrm{g}$ of aprotinin, $5 \mu \mathrm{g}$ of leupeptin, and $0.1 \mathrm{~mm}$ phenylmethylsulfonyl fluoride (PMSF)], and subject to immunoprecipitation (IP) with HA or DCC antibody (Ab). Bound biotin was detected with streptavidin-horseradish peroxidase (1:1000) in Western analysis.

Detection of bound netrin by immunofluorescence. Myc-tagged chicken netrin was concentrated from media (Optimem) of stably expressing HEK293 cells and purified on a heparin column (HiTrap Heparin HP; Amersham Biosciences, Arlington Heights, IL). Cos-7 cells plated on coverslips were transfected with DCC or Unc5c deletion constructs. The cells were incubated with myc-netrin at $1 \mu \mathrm{g} / \mathrm{ml}$ for $30 \mathrm{~min}$ before washing for $5 \mathrm{~min}$ in HBHA (20 mM HEPES, pH 7.0, and $0.5 \mathrm{mg} / \mathrm{ml} \mathrm{BSA}$ in HBSS; Invitrogen), rinsed with $1 \times$ PBS, and fixed in sequential steps with $4 \%$ paraformaldehyde in PBS for 5 min and 2\% paraformaldehyde and $0.2 \%$ Triton X-100 in PBS for 10 min. Fixed cells were washed three times with PBST (PBS with $0.1 \%$ Triton X-100), blocked with $100 \mathrm{~mm} \mathrm{NH}_{4} \mathrm{Cl}$ for $10 \mathrm{~min}$, washed three times with PBST, and blocked in PBST with $10 \% \mathrm{FBS}$ and $5 \%$ BSA for $30 \mathrm{~min}$. The primary antibodies, rabbit anti-HA or goat anti-DCC (Santa Cruz Biotechnology, Santa Cruz, CA) and mouse anti-myc (Covance, Princeton, NJ) were incubated for 45 min at 1:200 in PBST with 2\% FBS and 2\% BSA and washed three times for 5 min each with PBST. Secondary antibodies from Molecular Probes (Eugene, OR) (Alexa Fluor 488 goat anti-mouse and Alexa Fluor 594 donkey anti-rabbit for Unc5/netrin staining and Alexa Fluor 488 donkey anti-mouse and Alexa Fluor 594 donkey anti-goat for DCC/netrin staining) were incubated at 1:200 in PBST with 10\% FBS for $30 \mathrm{~min}$. After a final series of washes with PBST, coverslips were mounted on slides with Vectashield (Vector Laboratories, Burlingame, CA) and sealed with nail polish. Cells were imaged using a confocal microscope.

Unc5c tyrosine phosphorylation. HEK293 cells were transfected with HA-tagged Unc5c and deletion constructs with and without DCC. Twenty-four hours after transfection, cells were serum-starved overnight. Cells were washed with ice-cold PBS with $100 \mu \mathrm{M}$ pervanadate $\left(\mathrm{H}_{2} \mathrm{O}_{2}\right.$-activated sodium metavanadate) for $5 \mathrm{~min}$ and lysed in PLC buffer (50 mм HEPES, pH 7.5, $150 \mathrm{~mm} \mathrm{NaCl}, 1.5 \mathrm{~mm} \mathrm{MgCl}_{2}, 1 \mathrm{~mm}$ EGTA, $10 \mathrm{~mm}$ sodium pyrophosphate, $100 \mathrm{~mm} \mathrm{NaF}, 1 \%$ Triton X-100,
$10 \%$ glycerol, $1 \mathrm{~mm}$ Na orthovanadate, $5 \mu \mathrm{g}$ of aprotinin, $5 \mu \mathrm{g}$ of leupeptin, and $0.1 \mathrm{~mm}$ PMSF). Lysates were subject to IP using HA Ab. PY20 Ab (PharMingen, San Diego, CA) at 1:1000 dilution was used to detect tyrosine-phosphorylated Unc5c-HA in Western analysis. For netrin stimulation, cells were incubated with purified chick myc-netrin in the amounts indicated for 10 min before pervanadate treatment.

Caenorhabditis elegans transgenics. Nematodes were cultured by standard techniques. C. elegans strains were obtained from the C. elegans Genetics Stock Center and grown at $20^{\circ} \mathrm{C}$. Germ-line transformation of nematodes was performed using standard methods (Mello and Fire, 1995). Healthy hermaphrodites were injected with a mixture of Unc5 constructs at $100 \mathrm{ng} / \mu \mathrm{l}$ and sur-5::GFP plasmid (Yochem et al., 1998) at $10 \mathrm{ng} / \mu \mathrm{l}$ as a marker. Worms expressing the UNC-5 transgenes from extrachromosomal arrays were crossed with unc-5(e53). In both wt and unc-5(e53) backgrounds, worms expressing UNC-5 transgenes were assessed for uncoordination in forward and backward movement and DTC migration abnormalities by microscopic analysis of gonad morphology. Three independently analyzed batches $(n=30)$ for each worm strain were assessed. Statistical analysis was done using Statview software.

RNA was prepared for each transgenic strain from 20 young hermaphrodites collected in $50 \mu \mathrm{l}$ of GITC buffer ( $4 \mathrm{M}$ guanidinium isothiocyanate, $50 \mathrm{~mm}$ Tris- $\mathrm{HCl}$, pH 7.4, $50 \mathrm{~mm}$ EDTA, and 1\% Sarkosyl) frozen at $-70^{\circ} \mathrm{C}$ and thawed at $65^{\circ} \mathrm{C}$. Samples were dried and resuspended in $250 \mu$ l of Trizol (Invitrogen) and extracted according to the manufacturer's protocol. RT-PCR using the Titan One Tube RT-PCR system (Roche) was used to detect transgenic UNC-5 and actin transcripts. The ratio of UNC-5/actin was computed using NIH Image software.

For Western analysis, 15 worms expressing GFP throughout were collected and boiled for $10 \mathrm{~min}$ in $20 \mu \mathrm{l}$ of $2 \times$ Laemmli loading buffer, separated by SDS-PAGE, and transferred to nitrocellulose for Western blot analysis. Detection of UNC-5-HA required the use of Super Signal West Dura extended duration substrate (Pierce)

\section{Results}

Netrin-AP binds to Unc5- or DCC-expressing Cos-7 cells and is blocked by competing netrin

Netrin-AP was incubated with Unc5c- and DCC-expressing Cos-7 cells. Postfixation bound netrin was detected using nitroblue-tetrazolium-chloride-5-bromo-4-chlor-indolyl-

phosphate substrate. Dark staining cells indicating bound netrin are observed with DCC or Unc5 transfection but not with the pCDNA3 negative control (Fig. $1 A$ ). This binding can be blocked with preincubation of the cells with myc-netrin. No binding is detected with an irrelevant AP-tagged protein Semaphorin 4D.

Purified myc-tagged chicken netrin also binds to DCC- or Unc5c-expressing cells (Fig. $1 \mathrm{~B}$ ). Confocal imaging shows colocalization of netrin with both DCC and Unc5c. Cell surface expression of Unc5c and DCC is also evident.

\section{Unc5c requires the second Ig domain to bind netrin}

Unc5c has two Ig domains and two thrombospondin (Tsp) domains (Fig. 2A). Unc5c-HA deletion constructs were expressed in Cos-7 cells and incubated with soluble netrin-AP (Fig. $2 B$ ). Cell staining shows that deletion of either or both Tsp domains ( $\Delta$ Tsps) does not eliminate netrin binding, suggesting primary binding is with the Ig domains. Indeed, deletion of both Ig domains ( $\Delta$ Igs) abolishes binding. Deletion of either Ig domain causes a loss of binding at $2 \mathrm{nM}$ netrin, however when assayed with $5 \mathrm{~nm}$ netrin minimal staining is observed with $\Delta \operatorname{Ig} 1$ but not $\Delta$ Ig2 (Fig. $2 B$ ), suggesting that Ig2 contributes more to binding than Ig1. Cell surface expression of Unc 5 c receptor mutants was confirmed using a membrane impermeable biotinylation reagent to label cell surface proteins (Fig. 2C). Results indicate that all recombinant proteins described here are surface-expressed.

Confocal microscopy shows netrin bound to Unc5c- and $\Delta$ Tsps-expressing Cos-7 cells, but not cells expressing $\Delta$ Ig1 or 


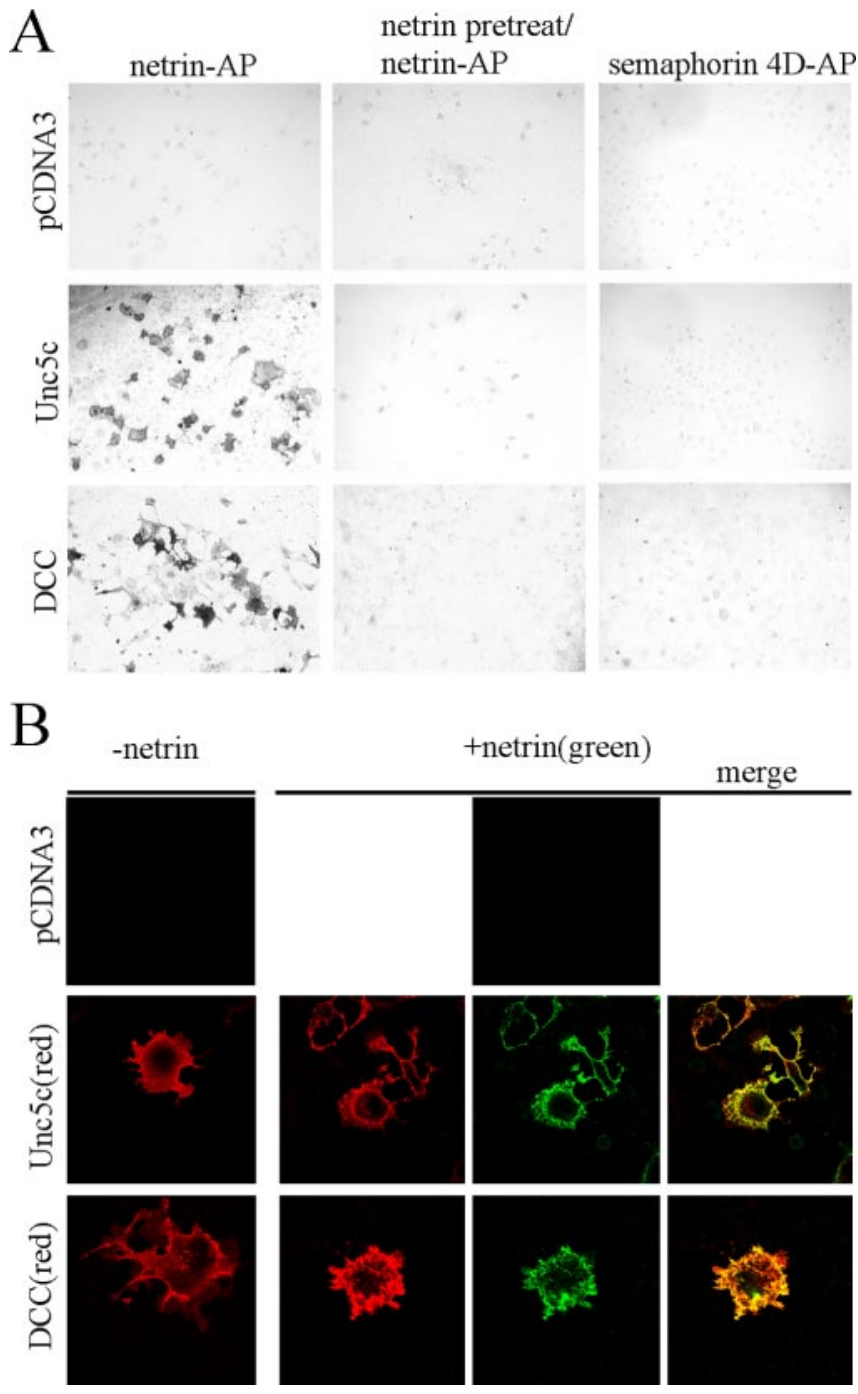

Figure 1. A, Binding of netrin-AP to Unc5C- and DCC-expressing Cos-7 cells, but not pCDNA3 transfected cells (left column). Preincubation of cells with purified chicken netrin blocks netrinAP binding (middle column). No binding is observed between semaphorin 4D-AP and DCC- and Unc5c-expressing cells (right column). B, Colocalization of myc-netrin bound to DCC- and Unc5expressing Cos-7 cells by immunofluorescence confocal microscopy. Examples of netrin-bound cells are shown for Unc5 (second row) and DCC (third row) with and without incubation of netrin before fixation. Unc $5 \mathrm{C}$ and $D C C$ (red) show surface expression. Bound netrin (green) is seen at the surface of DCC- or Unc5c-expressing cells only. No netrin binding is observed to untransfected cells. The merged image (last column) shows colocalization (yellow) of netrin bound to its receptors.

$\Delta$ Ig2 (Fig. 3). All Unc5c constructs show evidence of surface localization in immunostained cells. As noted before, binding to $\Delta \operatorname{Ig} 1$ was clear but close to the sensitivity threshold for the netrin-AP binding assay. Because binding to $\Delta \operatorname{Ig} 1$ is not seen in immunostaining, it suggests that the AP-netrin binding assay has greater sensitivity. One distinct advantage of the AP binding assay is the ability to carefully regulate exposure in the staining buffer to clearly differentiate background from stained cells.

A quantitative comparison of netrin binding using immunoprecipitated $w t$ and mutant Unc5c proteins in an on-bead binding assay (data not shown) gives results similar to those seen in cell staining. In the on-bead assay full-length Unc5c and $\Delta$ Tsps show comparable binding kinetics, indicating a $K_{\mathrm{d}}$ for human Unc5c of $\sim 15 \mathrm{~nm}$, similar to other mammalian Unc5 proteins (Leonardo et al., 1997). Nevertheless, both confocal microscopy
A

netrin

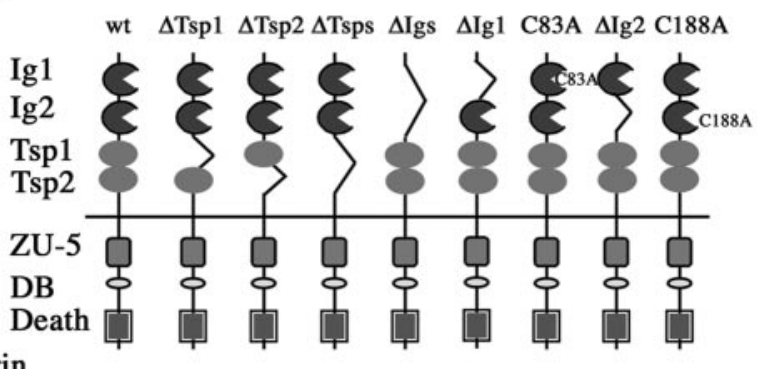

binding: $+++++++++++-\quad+/-\quad+-$ -

B

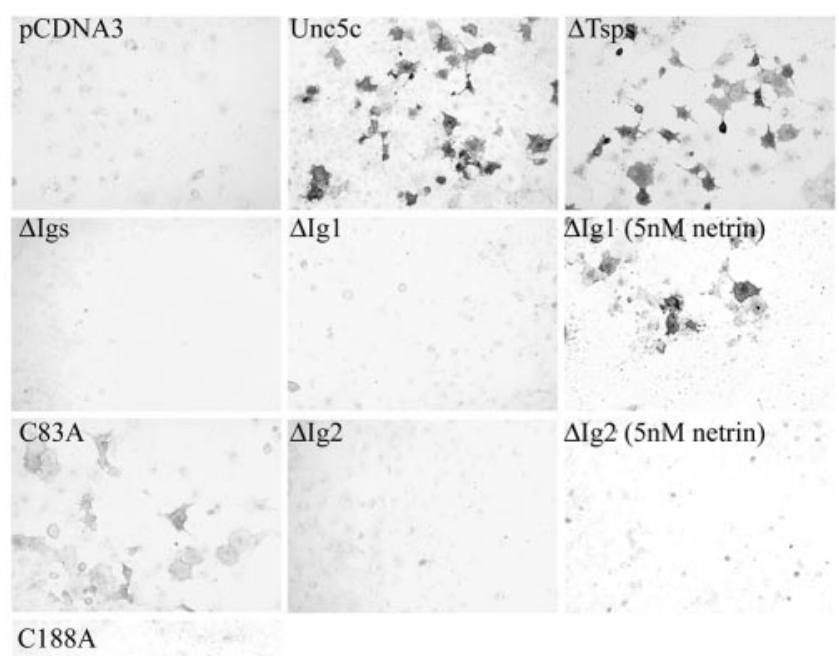

C

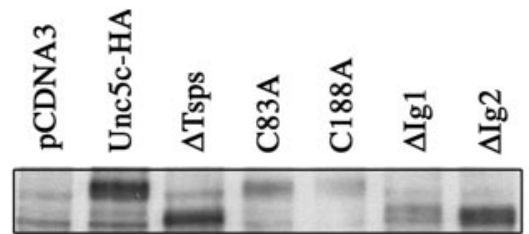

IP:HA

IB:streptavidin

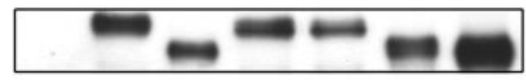

IP:HA

IB:HA

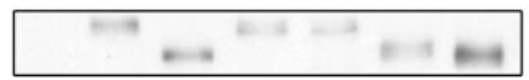

lysate

IB:HA

Figure 2. A, Unc5 has two Ig domains, two Tsp domains, a ZO-1/Unc5 (ZU-5) domain, a DCC binding motif (DB), and a death domain. Binding is summarized as follows to Unc5c and deletion constructs: $(+++)$ strong, $(+)$ weak, $(+/-)$ detectable above background at $5 \mathrm{~nm}$, and $(-)$ none detected. $B$, Binding of Unc $5 \mathrm{C}$ and deletion mutant expressing Cos-7 cells to netrinAP. Dark cells are Unc5c-expressing cells that have bound netrin-AP.C, Cell surface expression of Unc5c ectodomain deletions and point mutants. The top panel shows biotinylated proteins in the IP, the second shows protein in the IP, and the third protein in the lysates. All proteins show incorporation of the cell-impermeable biotinylation reagent.

and AP staining show somewhat diminished netrin binding to $\Delta$ Tsps than full-length, indicating some contribution for the Tsps domains to binding in the cellular context. A qualitative summary of the Unc5c binding to netrin is shown in Figure $2 \mathrm{~A}$. 

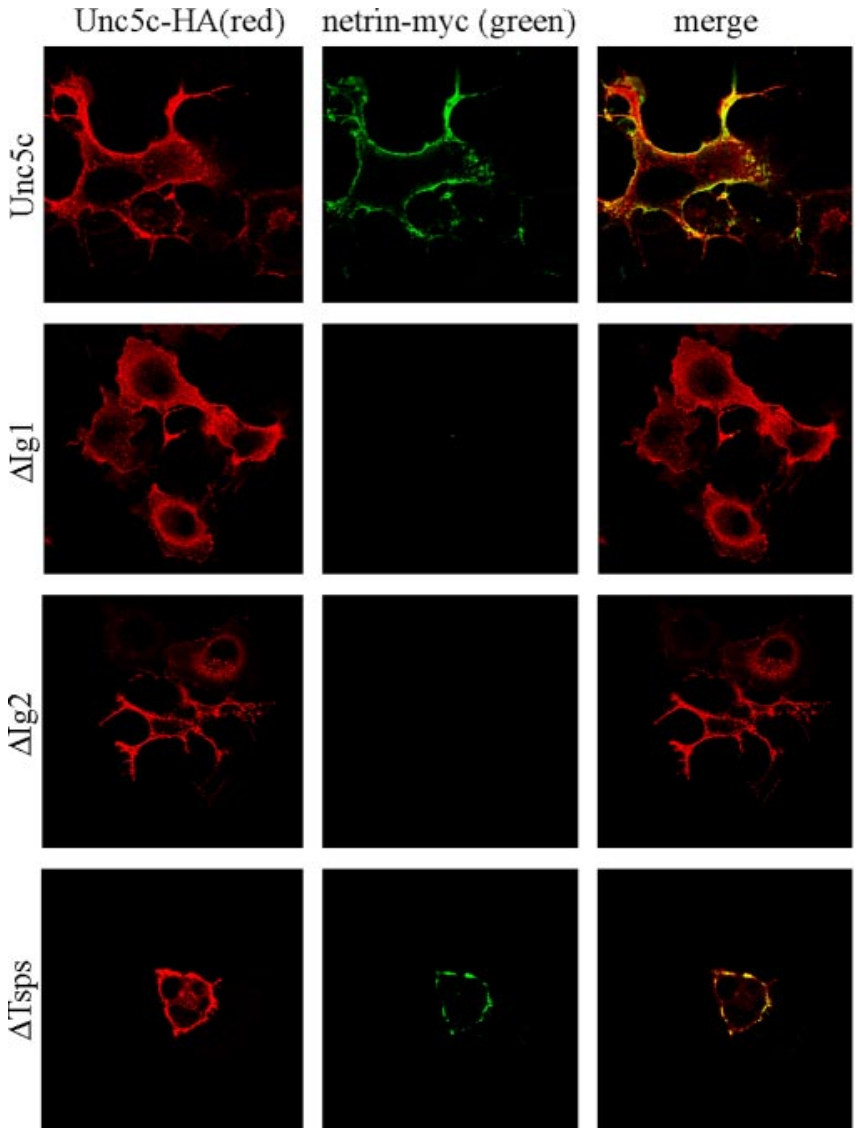

Figure 3. Binding and colocalization of Unc $5 \mathrm{c}$ and deletion mutants to myc-tagged netrin. Red indicates Unc5 expression. Green shows netrin binding. The merged image shows colocalization of netrin to Unc $5 \mathrm{C}$ and Unc $5 \mathrm{c}$ deletion constructs. The results show a pattern similar to netrin-AP staining (Fig. 2B).

The Ig domains in Unc5 share highest homology with the first two Ig domains of Robo, an axon guidance receptor that binds the ligand Slit (Li et al., 1999). To assess specificity of netrin binding to Unc5c, the Unc $5 c \Delta$ Igs expression plasmid was mutagenized at L52-P53 to create an NheI site into which cDNA for the two Ig domains of Robo (P29-V199) or Unc5c(P62-V257) was inserted. Although the Unc5c Ig domains restore binding in the netrin-AP cell-staining assay, the homologous region of Robol cannot, nor is binding observed between full-length Robol and netrin-AP (data not shown). Recently, it was shown that Robo Ig1 and Ig2 are critical for Slit binding (Liu et al., 2004). Even though Ig1 and Ig2 of Robo and Unc5c share homology and the ability to confer repulsive growth through ligand binding, netrin binding is unique to Unc5c.

\section{FN4 is necessary and sufficient to bind netrin}

DCC has four Ig domains and six fibronectin type III (FN) domains (Fig. 4A). To determine the domains in DCC necessary for netrin binding, ectodomain deletion constructs of human DCC were created, transfected into Cos-7 cells, and stained with netrin-AP as described (Fig. $4 B$ ). Deletion of FN3 through FN6 eliminates binding. FN5 has been previously implicated in netrin binding either directly or through an interaction mediated by heparin (Bennett et al., 1997; Geisbrecht et al., 2003). However, in our assay, deletion of FN5 does not abolish binding. DCC with deletion of both FN5 and FN6 ( $\Delta$ FN5-6) also

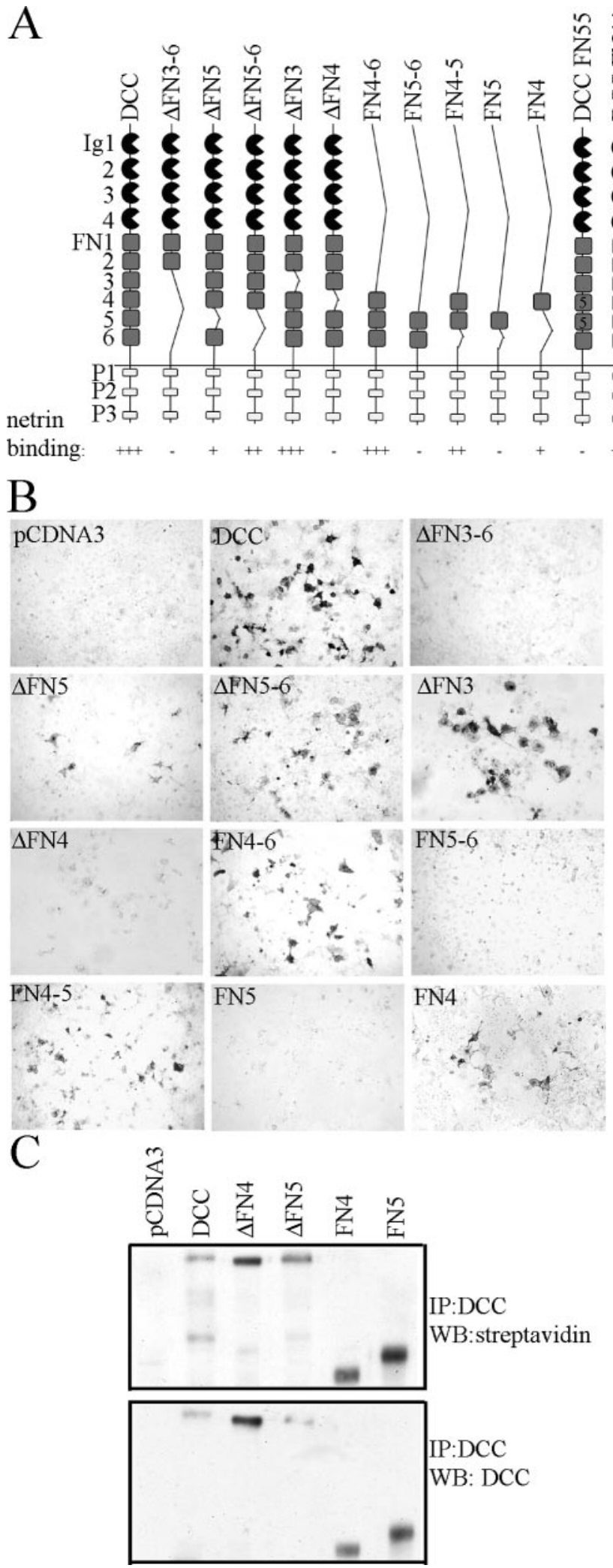

Figure 4. A, DCC has four Ig domains, six fibronectin type III (FN), and three conserved motifs $P 1, P 2$, and P3. Binding to DCC and deletion mutants is summarized as follows: $(+++)$ strong, $(++)$ moderate, $(+)$ weak, and $(-)$ none detected. $A$, B, Binding of DCC-expressing Cos-7 cells to netrin-AP. C, Evidence of surface expression of DCC deletion constructs. Cell surface expression is indicated by the incorporation of a cell-impermeable biotinylation reagent as detected by streptavidin-HRP in Western analysis. All constructs show evidence of surface expression. 

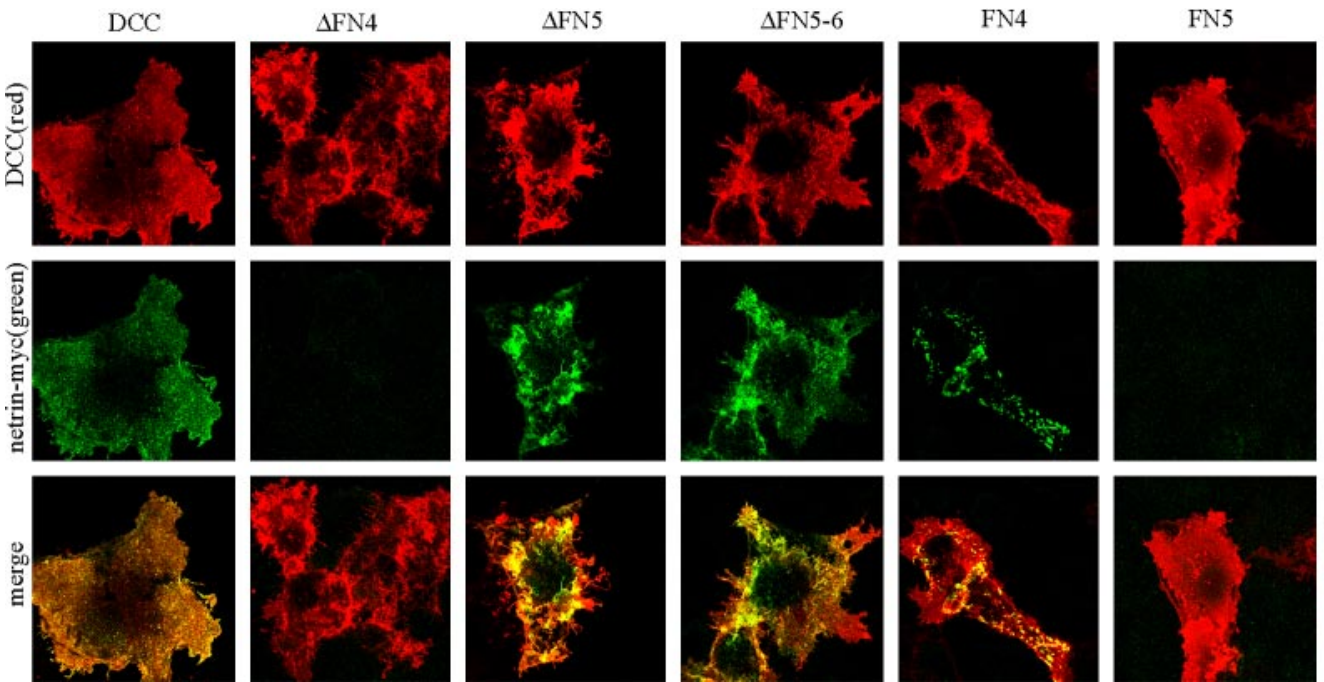
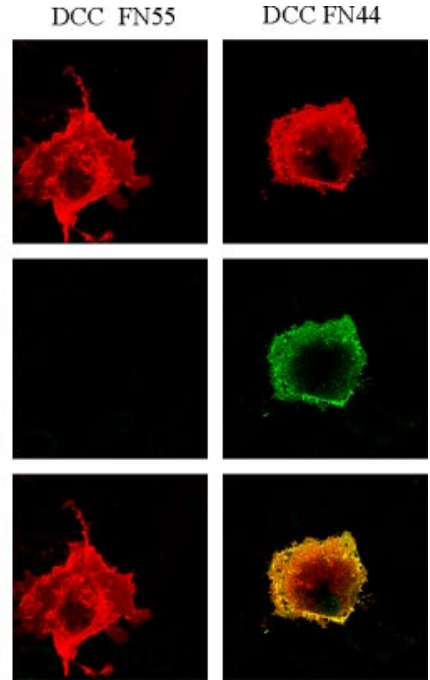

Figure 5. Binding and colocalization of DCC and deletion mutants with myc-tagged netrin. Red indicates DCC expression. Green shows bound netrin. The merged image shows colocalization of netrin with DCC and deletion constructs. The results show the same pattern of binding as with netrin-AP staining (Fig. $4 B$ ).

shows moderate staining, indicating a primary binding site with either FN3 or FN4.

Deletion of FN3 does not alter netrin binding, whereas deletion of FN4 eliminates binding. To see if FN4 is sufficient for netrin binding, an additional series of deletion mutants were created to isolate FN4 and FN5. Consistent with the preceding results, a deletion mutant with only the last three FN domains (FN4-6) shows strong binding, whereas FN5-6 does not (Fig. $2 A$ ). In a construct with FN4 and FN5 expressed together (FN4$5)$, moderate staining is observed. No netrin binding is detected when FN5 is expressed by itself, whereas when FN4 is expressed by itself, binding is seen. Cell surface expression of the most relevant constructs was confirmed using a cell-impermeable biotinylation reagent to label surface proteins. (Fig. 4C)

Immunofluorescence confocal microscopy was used to colocalize netrin with DCC (Fig. 5). As with the netrin-AP assay, myc-netrin bound to DCC, $\Delta \mathrm{FN} 5, \Delta \mathrm{FN} 5-6$, and FN4, but not $\Delta$ FN4 or FN5 only. Binding to FN4 was weak and distributed in puncta, possibly suggesting that DCC FN4 is internalizing bound netrin more rapidly than the other constructs or that the netrin binding elsewhere is below the detection threshold.

A previous report has suggested that the interaction with netrin is mediated by the FN5 domain of DCC. To more fully address this conflict, DCC constructs in which FN4 is substituted with FN5 (DCC FN55) or FN5 is substituted with FN4 (DCC FN44) were made as described in Materials and Methods and tested for netrin binding by immunostaining and confocal imaging. Binding is seen for DCC with two FN4 domains, but not with DCC with two FN5 domains (Fig. 5). Whereas some contribution of FN5 is possible because FN44 does not bind as well as wt DCC, direct evidence for an interaction between netrin and FN5 is not seen in our assays. A qualitative summary of netrin binding to DCC is shown in Figure 4A. Our data demonstrate that the FN4 domain is critical for netrin binding.

\section{Netrin binds its receptors via multiple domains}

Netrin has a Laminin VI-like (Lam VI) domain, a Laminin V-like (Lam V) domain, and a C-terminal domain (C345C) with homology to complement factors C3, C4, and C5 (Fig. 6A). Binding was assessed to DCC- and Unc5c-expressing Cos-7 cells using the cell staining assay with netrin-AP deletion mutants (Fig. 6B).
$\Delta$ Lam VI, $\Delta$ Lam V, and $\Delta$ C345C each bind DCC and Unc5c, indicating that no single domain in netrin is essential for binding to either receptor. Expression of individual netrin domains met with mixed results. Neither the Lam VI domain nor the Lam V domain could be expressed by itself despite our efforts with numerous constructs. However, a C345C-AP protein was made and found to bind to Unc5c- but not DCC-expressing cells. The C345C domain had been thought to be dispensable for DCC and Unc5 binding, although it has recently been shown to regulate adhesion and migration of epithelial cells through binding to the integrins $\alpha 6 \beta 4$ and $\alpha 3 \beta 1$ (Yebra et al., 2003). The binding of $\mathrm{C} 345 \mathrm{C}$ to Unc5c is weaker than that seen for full-length netrin and best observed at $5 \mathrm{nM}$. A qualitative summary of the binding of netrin mutants is shown in Figure $6 \mathrm{~A}$.

To further substantiate our view that FN4 is the primary domain in DCC necessary for netrin binding, we assessed binding between DCC deletions and the netrin deletion constructs $\Delta$ Lam VI, $\Delta \mathrm{Lam} \mathrm{V}$, and $\Delta \mathrm{C} 345 \mathrm{C}$. For each of these netrin construct binding was observed (Fig. $6 \mathrm{C}$ ), indicating that at least two regions (Lam VI and Lam V) of netrin bind to DCC FN4. Figure 7 shows a model for netrin, Unc5, and DCC interactions integrating information derived from this work and previous studies (Lim and Wadsworth, 2002; Geisbrecht et al., 2003).

\section{The Unc5 second Ig domain is autoinhibitory for} tyrosine phosphorylation

Tyrosine phosphorylation of Unc5 is stimulated by netrin in the presence of DCC and is important for the transduction of repulsive signals (Tong et al., 2001; Killeen et al., 2002). Consequently, tyrosine phosphorylation is assumed to be an indicator of activity. We wished to assess the consequences of deleting the netrin binding or netrin nonbinding regions of the Unc $5 \mathrm{c}$ ectodomain on Unc5c signaling. HA-tagged Unc5c, $\Delta \mathrm{Ig} 2$, and $\Delta$ Tsps were expressed in HEK293 cells and immunoprecipitated. Basal receptor tyrosine phosphorylation was measured by Western analysis using a phosphotyrosine antibody (Fig. 8). Both DCC coexpression and netrin stimulation increase Unc5c tyrosine phosphorylation (Fig. $8 A$ ). Deletion of the netrin binding domain $(\Delta \operatorname{Ig} 2)$ causes a significant increase in basal receptor tyrosine phosphorylation similar to DCC coexpression or netrin stimulation, whereas deletion of the netrin nonbinding region $(\Delta$ Tsps) shows 


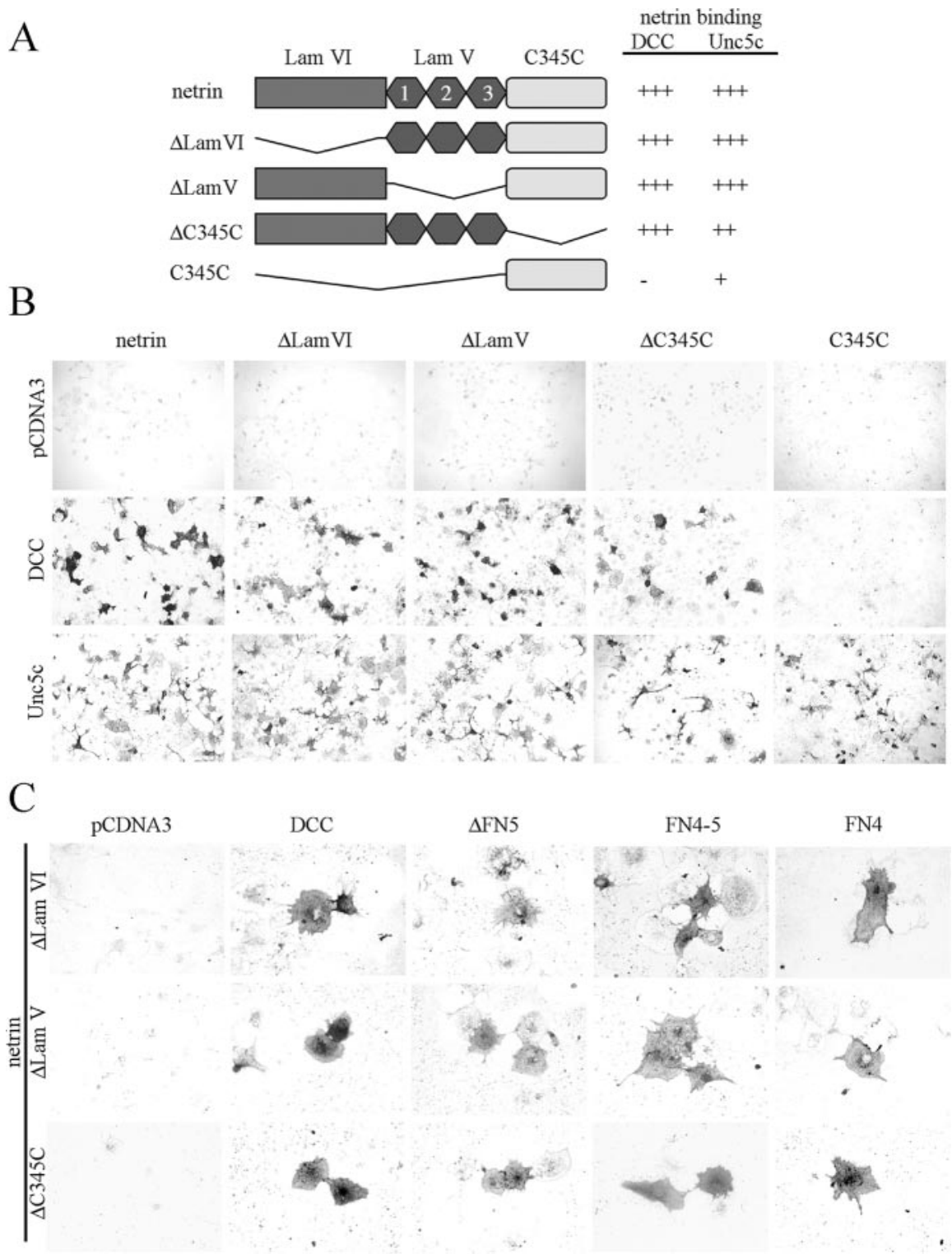

Figure 6. A, Netrin has three domains, a Laminin VI-like domain, three EGF repeats constituting a Laminin V-like domain, and a C-terminal C345C domain. Qualitative assessment of binding with netrin and deletion constructs is as follows: $(+++)$ strong, $(++)$ moderate, $(+)$ weak, and $(-)$ none detected. $B$, Binding of DCC- and Unc5c-expressing Cos-7 cells to netrin-AP deletion mutants. $C$, Netrin-AP deletion constructs binding to DCC deletions shows that multiple netrin domains interact with DCC through FN4. Because these are weak interactions, individual netrin-bound cells are shown. All netrin deletion constructs show binding to $\Delta$ FN5 and FN4. FN4 -5 does not differ from the binding seen to FN4.

decreased phosphorylation compared with $w t$ (Fig. $8 B$ ). With coexpression of DCC, no difference in tyrosine phosphorylation is observed between Unc5c, $\Delta \operatorname{Ig} 2$, and $\Delta$ Tsps. Thus, the difference seen with and without DCC coexpression is not attributable to a general inability of the Unc 5 c deletion mutants to become activated (because of variance in protein trafficking, misfolding, or other factors). Thus, the second Ig domain appears to autoinhibit Unc5c tyrosine phosphorylation. The Tsp domains help maintain $w t$ levels of basal tyrosine phosphorylation even though they are unnecessary for netrin binding.

\section{Functional analysis in C. elegans of removal of the netrin binding and nonbinding domains of UNC-5}

To assess the effect of eliminating the netrin binding Ig domains and nonbinding Tsp domains of UNC-5 in vivo, transgenic worms were created expressing UNC-5, UNC-5 $\Delta$ Igs, and UNC-5 $\Delta$ Tsps from extrachromosomal arrays. Relative expression of UNC-5 transgenes was determined using RT-PCR and Western analysis (Fig. $9 A, B)$. For each transgene, low and high expressing strains were generated.

unc-5(e53) worms display uncoordinated movement and have a high frequency of malformed gonads stemming from the absence of a ventral-dorsal migration of DTCs (Fig. 9C-E) Neither UNC-5 $\Delta$ Igs nor UNC-5 $\Delta$ Tsps rescues unc-5(e53) uncoordination or gonad defects, whereas UNC-5 expressed at the lower level provides substantial rescue for both phenotypes. We found that $86.1 \%$ of UNC-5 (low) expressing worms had improved forward movement, compared with no detectable improvement for either strain expressing UNC-5 $\Delta$ Igs (high) or UNC-5 $\Delta$ Tsps (high). Similar results were obtained with UNC-5 $\Delta$ Igs (low) and UNC-5 $\Delta$ Tsps (low). UNC-5 (low) also decreases the percentage of DTCs that fail to make a ventral to dorsal migration (Fig. $9 D, E)$. Rescue of the DTC migration defect is not observed for either UNC-5 $\Delta$ Igs or UNC-5 5 Tsps. These results indicate that both netrin binding and nonbinding domains are essential for UNC-5 function. Because UNC-5 (high) also does not rescue the DTC migration defects, it is clear that $u n c-5(e 53)$ rescue is highly sensitive to transgene expression level. Moreover, overexpression of UNC-5 (high and to a lesser degree, low) causes uncoordination in the wild-type background. The penetrance of this effect varies between transgenes affecting $100 \%$ of UNC-5 (high), $74.1 \pm 4.3 \%$ of UNC- $5 \Delta$ Igs (high), and $18.1 \pm 4.1 \%$ of UNC- $5 \Delta$ Tsps (high) expressing worms.

At the higher level of expression, UNC-5 partially rescues forward movement $(33.3 \%)$, but does not restore normal gonad morphology. Instead, abnormalities are frequently observed along the ventral to dorsal segment of the DTC migration pathway in both $w t$ and unc-5(e53) worms. In these transgenic worms the gonad arms are usually club-shaped rather than U-shaped (Fig. 8C-E). During the second phase of migration, instead of going directly across, the DTC is misdirected, ultimately reaching the dorsal side at a position in a plane of focus deeper than normal. Likewise the third phase is altered and truncated. This phenotype differs from the other previously reported phenotype for UNC-5 overexpression in which premature expression of UNC-5 driven by the emb-9 promotor causes an early turning phenotype (Su et al., 2000). The difference between that phenotype and the one described here is likely attributable to the fact that the UNC-5 construct we use is driven by the endogenous promotor (Killeen et al., 2002).

Because the DTC migration defect is different from that re- 


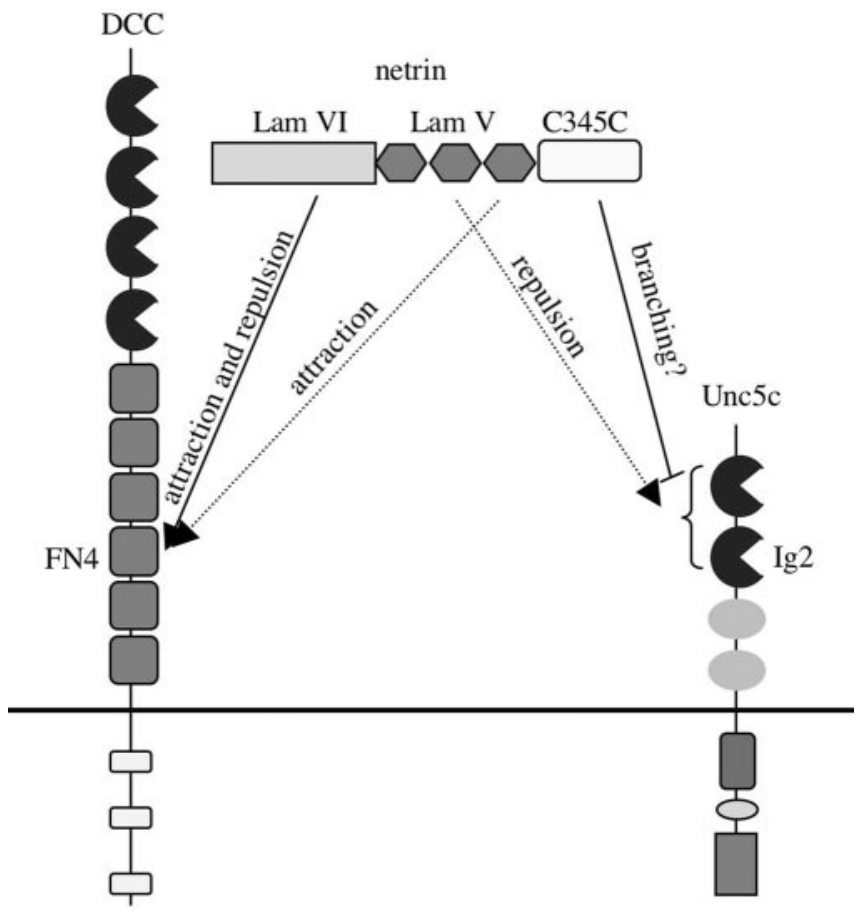

Figure 7. A model of Unc5, DCC, and netrin interactions. Dotted lines denote inferences from the work of Lim and Wadsworth (2002) regarding particular netrin EGF-like repeats involved in receptor binding. Critical domains in DCC (FN4) and Unc5 (Ig2) are indicated. A bracket is used to note Ig1 of Unc5, which also contributes to netrin binding, but is insufficient by itself to bind netrin in our assays.

sulting from the UNC-5 loss of function mutation, we assume that these effects are a novel gain-of-function likely caused in part by an excess of UNC-5 signal that is seen in both $w t$ and $u n c$ 5(e53) genetic backgrounds. Consequently, we generated a phosphorylation site mutant (Y482F) previously shown to be essential for repulsive signaling (Killeen et al., 2002) and compared it to the UNC-5 line expressing at the lower level. In the wt background, UNC-5 (Y482F) expression produces neither clubshaped gonads nor uncoordination. For UNC-5 (low) worms, the frequencies of uncoordination and club-shaped gonads were was $16.8 \pm 2.0$ and $7.2 \pm 0.5 \%$, respectively. These results indicate that uncoordination and the club-shaped gonad phenotype seen with UNC-5 overexpression is at least partially dependent on Y482. As with phenotypic rescue, the penetrance of this gainof-function phenotype is dependent on both netrin binding and nonbinding domains. The likelihood of club-shaped gonads is lower in UNC-5 $\Delta$ Igs (high) and UNC-5 $\Delta$ Tsps (high) than in UNC-5 (high) expressing line (Fig. 9D,E).

\section{Discussion}

This study determines the domains required for the interactions between netrin and the axon guidance receptors Unc5c and DCC in a cellular context. We explored the role of the netrin-binding domain in Unc5 in cultured cells and conclude that it is autoinhibitory for receptor phosphorylation. Furthermore, our data show that the netrin nonbinding region, the Tsp domains, is also important for Unc5 function in vitro and in C. elegans.

\section{Unc5 binding to netrin}

Both Unc5c Ig domains are necessary for full netrin binding. Our cell-based binding assays, which are similar to physiological conditions of netrin binding cell surface receptors, indicate that al-
A

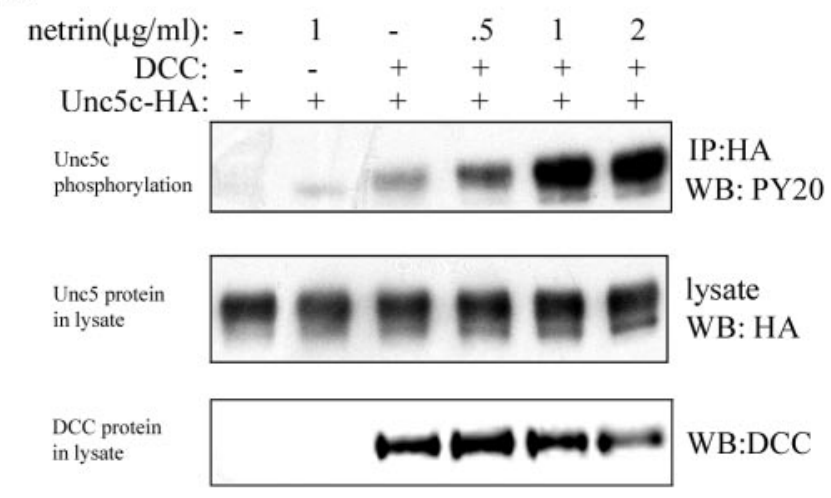

B

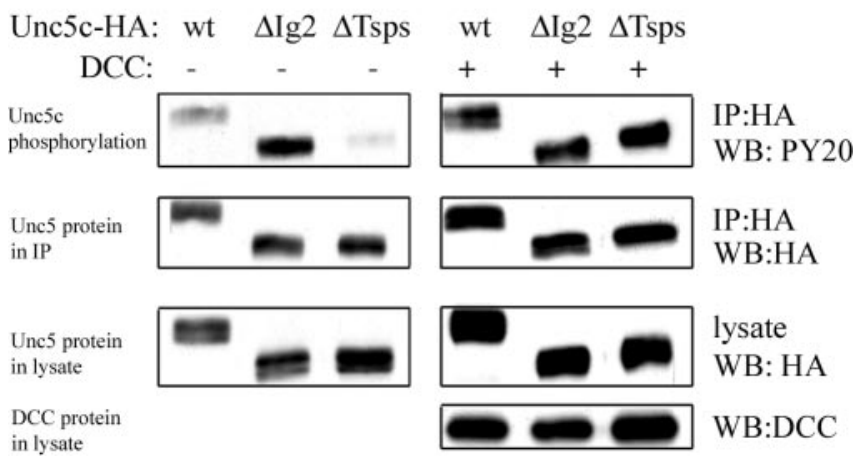

Figure 8. Basal and netrin-stimulated tyrosine phosphorylation of Unc 5 c in HEK293 cells. A, Phosphorylation of full-length Unc 5 c. Unc 5 c phosphorylation is stimulated by netrin and augmented by DCC coexpression. DCC coexpression and netrin stimulation have additive effects. $B$, Phosphorylation of Unc $5 \mathrm{c}$ and deletion constructs. $\Delta \mathrm{lg} 2$ shows more basal tyrosine phosphorylation, and $\Delta$ Tsps shows less tyrosine phosphorylation than full-length Unc $5 \mathrm{c}$. The Unc $5 \mathrm{c}$ deletion constructs show similar phosphorylation when DCC is coexpressed.

though both Ig domains contribute to netrin binding, the Ig domains are not redundant. Deletion of Ig1 reduces netrin binding, whereas deletion of Ig2 abolishes it. A previous study using a combination of bacterial and cell line-expressed recombinant proteins in in vitro binding assays (Geisbrecht et al., 2003) had found that both Ig domains of Unc5 bound immobilized netrin lacking its C345C domain, but did not address the issue of Ig domain redundancy. Moreover, by using full-length netrin we identified an additional interaction between the C345C domain and Unc5c.

Deletion of the netrin-binding domain in Unc5c leads to an increase in receptor tyrosine phosphorylation in vitro. Precedence for the relief-of-inhibition mode of axon guidance receptor signaling comes from studies on the plexin A1 receptor in which deletion of the Sema domain causes constitutive activation (Takahashi and Strittmatter, 2001). It has been previously shown that netrin binding stimulates tyrosine phosphorylation of the rostral cerebellar malformation protein (RCM), a mammalian homolog of UNC-5, and recruits the binding of Shp2 a tyrosine phosphatase (Tong et al., 2001) where it may modulate RCM signaling directly or indirectly through unknown downstream signaling molecules.

\section{DCC binding to netrin}

In this study, the FN4 domain of DCC is necessary and sufficient for netrin binding. Previous work has suggested that FN5 partic- 


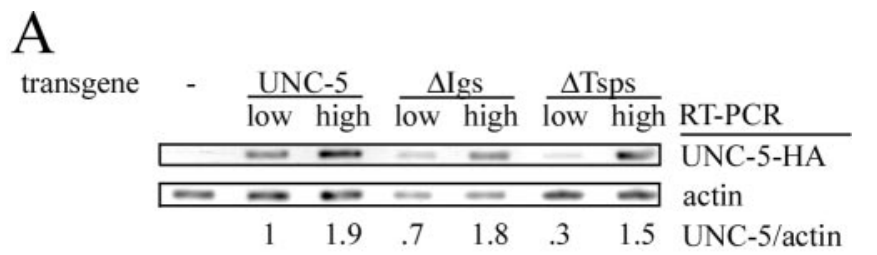

B
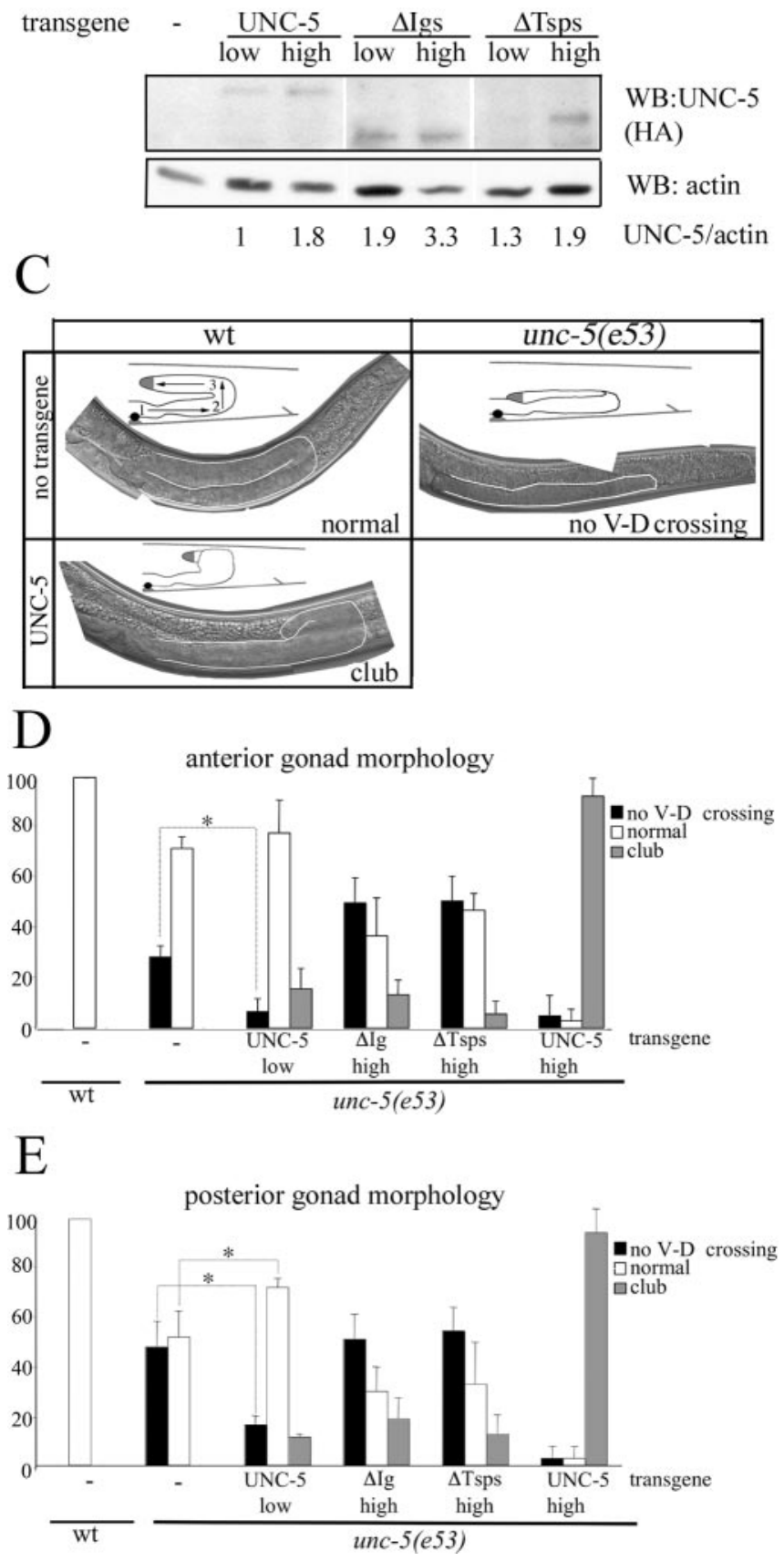

Figure 9. A, RT-PCR from RNA purified from nematodes expressing UNC -5 transgenes. The intensity of the transgenic UNC-5-specific band compared with the actin control indicates the relative amounts of transcription of each transgene. A reverse primer embedded in the HA tag was used to ensure transgene specificity. $B$, Fifteen transgenic worms for each strain were collected and analyzed for UNC-5-HA expression by Western blot analysis. The ratio of UNC -5 to actin is calculated. C, Images showing the posterior gonad arm in wt and unc-5(e53) worms. In wt worms, U-shaped gonad arms result from the migration of DTCs. The three phases of this migration in wt and unc -5 worms are depicted in the diagram inserts with numbered arrows. The second phase of this migration, in which DTCs move from the ventral side to the dorsal side, is dependent on UNC-6/netrin, UNC-40/DCC, and UNC-5. Representative phenotypes are shown ipates in an interaction with netrin directly (Geisbrecht et al., 2003) or indirectly via heparin (Bennett et al., 1997). We are unable to show that FN5 is sufficient for binding within the context of a membrane-expressed protein, although deletion of FN5 does decrease binding (Figs. 4, 5, 6C). If a DCC-netrin interaction is partially mediated by heparin, competition with heparin used in our assays to block nonspecific binding could inhibit detection of those interactions. It has been shown that an antiDCC antibody that blocks netrin stimulation does not block netrin binding (Bennett et al., 1997), implying that more than one domain could be involved in binding and activation. For example, FN5 may contribute to binding, while FN4 is important for binding and activation, or vice versa. Although we cannot exclude FN5 as contributing to the netrin interaction, our observations indicate that both of the relevant domains of netrin ( $\mathrm{Lam} \mathrm{V}$ and Lam VI) bind to FN4 (Fig. 6C).

\section{Netrin binding to its receptors}

Netrin deletion mutants show that no single domain is required for binding to either Unc5 or DCC. With this information it is possible to compare our binding studies with the published findings in nematodes examining the effect of netrin domain deletions (Lim and Wadsworth, 2002). Experiments in nematodes show that the Lam VI domain is essential for both Unc6mediated attraction and repulsion (Lim and Wadsworth, 2002). We can deduce from our binding data that netrin has a high affinity binding site for DCC in the Lam VI domain (Fig. 7). Also, specific deletion of the LamV third EGF-like repeat causes ventral guidance defects similar to unc40 (Lim and Wadsworth, 2002). Consequently, we infer that this subdomain within the netrin LamV domain also has a high affinity DCC/Unc40 binding site, consistent with our binding data (Fig. 7).

Our results indicate that Unc5c binds to at least two regions of netrin. In worms, deletion of the second EGF-like domain causes disruption of dorsal axon guidance, similar to unc5 null mutants (Wadsworth et al., 1996), indicating a specific role for this region in Unc5 signaling. Although we do not exclude the possibility of an interaction between Unc5 and the Lam VI domain, it is simplest to propose that the role of the Lam VI domain in repulsion is mediated through its interaction with DCC and not Unc5 (Fig. 7).

The C345C domain suppresses branching of neurons from the ventral nerve cord in nematodes (Lim et al., 1999). Ectopic branching has also been shown to be Unc5- and Unc40dependent. Therefore, the binding we observe between Unc5 and the netrin $\mathrm{C} 345 \mathrm{C}$ domain could underlie a specific signaling complex regulating branching initiation. Because this interaction is relatively weak, a high concentration of netrin-Unc6 could be needed to initiate this complex. In fact, in nematodes branching suppression is known to occur at the ventral midline where

for the posterior gonad arm (outlined in white) for each wt, unc-5(e53), and a worm expressing the UNC -5 transgene at the higher expression level. In the unc-5(e53) panel, the DTC does not have a ventral to dorsal migration and returns to the vulval region in a different plane of focus than the first phase of migration. In all pictures, ventral is at bottom and posterior to the right. $D, E$, Morphologies of the anterior $(D)$ and posterior $(E)$ gonad arms with UNC -5 transgene expression. In the development of unc-5(e53) worms, DTCs frequently fail to have a ventral to dorsal migration (black bars). With transgenic expression of UNC -5 at the lower level this percentage decreases, indicating phenotypic rescue $\left(^{*} p<0.01\right)$. UNC $-5 \Delta$ lgs, UNC $-5 \Delta$ Tsps, or UNC-5 expressed at the higher level did not rescue the DTC migration defect. The club-shaped gain-of-function phenotype is also observed at different frequencies between constructs (gray bars). White bars indicate normal gonad formation. 
UNC-6 expression is highest. Available evidence also indicates that the $\mathrm{C} 345 \mathrm{C}$ domain needs simultaneous expression of the rest of netrin to suppress branching (Lim and Wadsworth, 2002; Wang and Wadsworth, 2002). Consequently, the C345C domain may link Unc5 with another Unc5 or DCC molecule to form a unique signaling complex that inhibits branching. Proving the existence and nature of such a complex would indicate additional flexibility in netrin signaling governed primarily through differential binding affinities of specific regions of netrin to its receptors.

\section{The role of the domains not involved in netrin binding}

Although each of the netrin domains has receptor interactions that correlate with known cellular events, the same cannot be said for the netrin receptors themselves. It is reasonable to wonder as to the purpose of the domains in DCC and Unc5 not involved in netrin binding. Here, more can be speculated about the Tsp domains of Unc5. Other studies, in addition to our own, have shown that the Tsp domains are functionally important in worms (Killeen et al., 2002) for phenotypic rescue of $u n c-5$ mutations and in flies (Keleman and Dickson, 2001) to produce the same gain-of-function phenotype as pan-neuronal expression of $w t$ Unc5.

Although the Tsp domains may have a role in promoting receptor tyrosine phosphorylation that is inhibited by the presence of the netrin binding Ig domains, the Tsp domains could also have a function independent of netrin-UNC-6 signaling. It has been suggested that UNC-129 may be another ligand for UNC-5 that binds via the Tsp domains (Colavita et al., 1998), although direct evidence of this has not been forthcoming. However, because deletion of the Ig domains appears to mimic netrin binding, it will be important to determine whether deletion of the Tsp domains mimics the activity of another factor that acts to decrease Unc5 tyrosine phosphorylation.

\section{References}

Bennett KL, Bradshaw J, Youngman T, Rodgers J, Greenfield B, Aruffo A, Linsley PS (1997) Deleted in colorectal carcinoma (DCC) binds heparin via its fifth fibronectin type III domain. J Biol Chem 272:26940-26946.

Chan SS, Zheng H, Su MW, Wilk R, Killeen MT, Hedgecock EM, Culotti JG (1996) UNC-40, a C. elegans homolog of DCC (Deleted in Colorectal Cancer), is required in motile cells responding to UNC-6 netrin cues. Cell $87: 187-195$

Colamarino SA, Tessier-Lavigne M (1995) The axonal chemoattractant netrin-1 is also a chemorepellent for trochlear motor axons. Cell 81:621-629.

Colavita A, Krishna S, Zheng H, Padgett RW, Culotti JG (1998) Pioneer axon guidance by UNC-129, a C. elegans TGF-beta. Science 281:706-709.

Dickson B (2002) Molecular mechanisms of axon guidance. Science 298:1959-1964.

Geisbrecht BV, Dowd KA, Barfield RW, Longo PA, Leahy DJ (2003) Netrin binds discrete subdomains of DCC and UNC5 and mediates interactions between DCC and heparin. J Biol Chem 278:32561-32568.

Hedgecock EM, Culotti JG, Hall DH (1990) The unc-5, unc-6, and unc-40 genes guide circumferential migrations of pioneer axons and mesodermal cells on the epidermis in C. elegans. Neuron 4:61-85.

Hong K, Hinck L, Nishiyama M, Poo MM, Tessier-Lavigne M, Stein E (1999) A ligand-gated association between cytoplasmic domains of UNC5 and DCC family receptors converts netrin-induced growth cone attraction to repulsion. Cell 97:927-941.
Ishii N, Wadsworth WG, Stern BD, Culotti JG, Hedgecock EM (1992) UNC-6, a laminin-related protein, guides cell and pioneer axon migrations in C. elegans. Neuron 9:873-881.

Keino-Masu K, Masu M, Hinck L, Leonardo ED, Chan SS, Culotti JG, TessierLavigne M (1996) Deleted in Colorectal Cancer (DCC) encodes a netrin receptor. Cell 87:175-185.

Keleman K, Dickson BJ (2001) Short- and long-range repulsion by the Drosophila Unc5 netrin receptor. Neuron 32:605-617.

Kennedy TE, Serafini T, de la Torre JR, Tessier-Lavigne M (1994) Netrins are diffusible chemotropic factors for commissural axons in the embryonic spinal cord. Cell 78:425-435.

Killeen M, Tong J, Krizus A, Steven R, Scott I, Pawson T, Culotti J (2002) UNC-5 function requires phosphorylation of cytoplasmic tyrosine 482, but its UNC-40-independent functions also require a region between the ZU-5 and death domains. Dev Biol 251:348-366.

Leonardo ED, Hinck L, Masu M, Keino-Masu K, Ackerman SL, TessierLavigne M (1997) Vertebrate homologues of C. elegans UNC-5 are candidate netrin receptors. Nature 386:833-838.

Li HS, Chen JH, Wu W, Fagaly T, Zhou L, Yuan W, Dupuis S, Jiang ZH, Nash W, Gick C, Ornitz DM, Wu JY, Rao Y (1999) Vertebrate slit, a secreted ligand for the transmembrane protein roundabout, is a repellent for olfactory bulb axons. Cell 96:807-818.

Lim YS, Wadsworth WG (2002) Identification of domains of netrin UNC-6 that mediate attractive and repulsive guidance and responses from cells and growth cones. J Neurosci 22:7080-7087.

Lim YS, Mallapur S, Kao G, Ren XC, Wadsworth WG (1999) Netrin UNC-6 and the regulation of branching and extension of motoneuron axons from the ventral nerve cord of Caenorhabditis elegans. J Neurosci 19:7048-7056.

Liu Z, Patel K, Schmidt H, Andrews W, Pini A, Sundaresan V (2004) Extracellular Ig domains 1 and 2 of Robo are important for ligand (Slit) binding. Mol Cell Neurosci 26:232-240.

Mello C, Fire A (1995) DNA transformation. Methods Cell Biol 48:451-482.

Su M, Merz DC, Killeen MT, Zhou Y, Zheng H, Kramer JM, Hedgecock EM, Culotti JG (2000) Regulation of the UNC-5 netrin receptor initiates the first reorientation of migrating distal tip cells in Caenorhabditis elegans. Development 127:585-594.

Takahashi T, Strittmatter SM (2001) Plexinal autoinhibition by the plexin sema domain. Neuron 29:429-439.

Tong J, Killeen M, Steven R, Binns KL, Culotti J, Pawson T (2001) Netrin stimulates tyrosine phosphorylation of the UNC-5 family of netrin receptors and induces Shp2 binding to the RCM cytodomain. J Biol Chem 276:40917-40925.

Vikis HG, Li W, Guan KL (2002) The plexin-B1/Rac interaction inhibits PAK activation and enhances Sema4D ligand binding. Genes Dev 16:836-845.

Wadsworth WG, Bhatt H, Hedgecock EM (1996) Neuroglia and pioneer neurons express UNC-6 to provide global and local netrin cues for guiding migrations in C. elegans. Neuron 16:35-46.

Wang Q, Wadsworth WG (2002) The C domain of netrin UNC-6 silences calcium/calmodulin-dependent protein kinase- and diacylglyceroldependent axon branching in Caenorhabditis elegans. J Neurosci 22:2274-2282.

Yebra M, Montgomery AM, Diaferia GR, Kaido T, Silletti S, Perez B, Just ML, Hildbrand S, Hurford R, Florkiewicz E, Tessier-Lavigne M, Cirulli V (2003) Recognition of the neural chemoattractant Netrin-1 by integrins alpha6beta4 and alpha3betal regulates epithelial cell adhesion and migration. Dev Cell 5:695-707.

Yochem J, Gu T, Han M (1998) A new marker for mosaic analysis in Caenorhabditis elegans indicates a fusion between hyp6 and hyp7, two major components of the hypodermis. Genetics 149:1323-1334. 